\title{
ON THE LATTICE THEORY OF IDEALS $\dagger$
}

\section{BY GARRETT BIRKHOFF}

1. Outline. The ideals of any ring define, relative to g.c.f. and 1.c.m., a combinatorial system having properties which we shall presently define as characterizing $B$-lattices.

In this article we shall first develop some new properties of $B$-lattices as abstract systems; the main results of this part of the work find expression in Theorems $1-5$. Then we shall apply this theory and some older results to the ideals of commutative rings $R$ which possess a principal unit $l$ and satisfy the Basis Theorem. In addition to developing the known theory of einartig ideals by combinatory methods, we give a necessary and sufficient condition that the $B$-lattice defined by the ideals of $R$ should be isomorphic with a ring of point sets in the sense of Hausdorff. $\ddagger$

2. Notation; Lattice Algebras. We shall in general use capital letters to denote systems, and small letters for elements. $a \in A$ will mean " $a$ is an element of the system $A$ "; $B \subset A$ will mean " $b \in B$ implies $b \in A$ " $B<A$ will mean $B \subset A$ but $B \neq A$.

By a lattice algebra will be meant any system $L$ which satisfies the following postulates:

(L1). Any $a \in L$ and $b \in L$ determine a unique "join" $a \mathrm{n} b \in L$ and a unique "meet" $(a, b) \in L$.

(L2). $a \cap b=b \cap a$ and $(a, b)=(b, a)$ for any $a \in L$ and $b \in L$.

(L3). $a \cap(b \cap c)=(a \cap b) \cap c$ and $(a,(b, c))=((a, b), c)$ for any $a \in L, b \in L$, and $c \in L$.

(L4). $a \cap(a, b)=a$ and $(a, a \cap b)=a$ for any $a \in L$ and $b \in L$.

From (L1)-(L4) follow $a \cap a=(a, a)=a$. Moreover $a \mathrm{n} b=b$ is equivalent to $(a, b)=a$; in this case we write $a \subset b$ or $b \supset a$, and $a \subset b$ taken with $b \subset c$ implies $a \subset c$. Moreover, $a<b$ means $a \subset b$ but $a \neq b$, while " $b$ covers $a$ " means $a<b$, but that no $x \in L$ satisfies $a<x<b$.

The reader may find it helpful to regard lattices as distorted

$\dagger$ Presented to the Society, March 30, 1934.

$\ddagger$ Hausdorff, Mengenlehre, 1927, p. 77. 
Boolean algebras in which $a \cap b$ is substituted for $a+b$, and $(a, b)$ for $a \cdot b$.

The following additional conditions are optional:

(L5). If $a \subset c$, then $a \cap(b, c)=(a \cap b, c)$.

(L6). $(a, b \cap c)=(a, b) \mathbf{n}(a, c)$ for any $a \in L, b \in L$, and $c \in L$.

If a lattice satisfies (L5), it is called a $B$-lattice; if it satisfies (L6), it is called a $C$-lattice. Any $C$-lattice is a $B$-lattice, and also satisfies $a \mathbf{n}(b, c)=(a \mathbf{n} b, a \mathbf{n} c)$.

3. Subdirect Decomposition. We shall consider in $\S \S 3-4$ only lattices $L$ which have a "largest" element $j$ satisfying $a \boldsymbol{n} j=j$ for every $a \in L$; such is always the case in applications. $\dagger$

We shall say that $a \in L$ and $b \in L$ are coprime if and only if $a \cap b=j$. We shall say that two sublattices $\ddagger A \subset L$ and $B \subset L$ are coprime if and only if $a \in A$ and $b \in B$ imply $a \cap b=j$. We shall say that the sublattices of a finite or transfinite $\$$ sequence of sublattices $A_{1} \subset L, \cdots, A_{n} \subset L$ are strongly coprime if and only if every $A_{i}$ is coprime with the sublattice generated by\| the other sublattices of the sequence.

Let $B_{1}, \cdots, B_{n}$ be any (finite or transfinite) sequence of lattices, whose largest elements are $j_{1}, \cdots, j_{n}$. By an $f$-type vector $\left[b_{1}, \cdots, b_{n}\right],\left(b_{i} \in B_{i}\right)$, we mean one in which $b_{i}=j_{i}$ except for a finite set of subscripts $i$. By the subdirect product $B_{1} \hat{x} \cdots \hat{x} B_{n}$ $=B^{*}$ of the $B_{i}$ is meant the lattice whose elements are the $f$-type vectors just defined, and such that by definition

$$
\begin{aligned}
{\left[b_{1}, \cdots, b_{n}\right] \cap\left[b_{1}^{\prime}, \cdots, b_{n}^{\prime}\right] } & =\left[b_{1} \cap b_{1}^{\prime}, \cdots, b_{n} \cap b_{n}^{\prime}\right], \\
\left(\left[b_{1}, \cdots, b_{n}\right],\left[b_{1}^{\prime}, \cdots, b_{n}^{\prime}\right]\right) & =\left[\left(b_{1}, b_{1}^{\prime}\right), \cdots,\left(b_{n}, b_{n}^{\prime}\right)\right] .
\end{aligned}
$$

$B^{*}$ is evidently a lattice with largest element $\left[j_{1}, \cdots, j_{n}\right]$. Further, if $B_{i}{ }^{*}$ denotes the sublattice of elements of the form $\left[j_{1}, \cdots, j_{i-1}, b_{i}, j_{i+1}, \cdots, j_{n}\right]$ of $B^{*}$, then $B_{i}^{*}$ is isomorphic with $B_{i}$, the lattices $B_{1}{ }^{*}, \cdots, B_{n}^{*}$ are strongly coprime, and any element of $B^{*}$ can be expressed as the meet of a finite num(L3.)

$\dagger$ In fact, if the number of elements of $L$ is finite, this follows from (L1)-

$\ddagger$ A sublattice $A$ of $L$ is any subsystem such that $a \in A$ and $a^{\prime} \in A$ imply $a \cap a^{\prime} \in A$ and $\left(a, a^{\prime}\right) \in A$.

$\S$ That is, in which the subscripts run through transfinite ordinals.

\| By the "sublattice generated by" is meant the least sublattice containing. 
ber of elements in the various $B_{i}{ }^{*}$. Finally, if the $B_{i}$ are $B$-lattices, then so $\dagger$ is $B^{*}$.

Conversely, let $B$ be any $B$-lattice, and let $B_{1}, \cdots, B_{n}$ be any finite or transfinite sequence of strongly coprime sublattices of $B$ such that any $b \in B$ can be expressed as the meet $\left(b_{i_{1}}, \cdots, b_{i_{m}}\right)$ of a finite number of $b_{i_{k}} \in B_{i_{k}}$.

For any $b \in B$ and $b^{\prime} \in B$ we can evidently so reorder the $B_{i}$ that $b=\left(b_{1}, \cdots, b_{m}\right), b^{\prime}=\left(b_{1}^{\prime}, \cdots, b_{m}{ }^{\prime}\right)$, and $b \cap b^{\prime}=b^{\prime \prime}$ $=\left(b_{1}^{\prime \prime}, \cdots, b_{m}^{\prime \prime}\right)$, where $b_{i} \in B_{i}, b_{i}^{\prime} \in B_{i}, b_{i}^{\prime \prime} \in B_{i}$, and $m$ is finite. But by (L2)-(L3), we have

$\left(b, b^{\prime}\right)=\left(\left(b_{1}, \cdots, b_{m}\right),\left(b_{1}^{\prime}, \cdots, b_{m}{ }^{\prime}\right)\right)=\left(\left(b_{1}, b_{1}^{\prime}\right), \cdots,\left(b_{m}, b_{m}{ }^{\prime}\right)\right)$.

Further if we set $a_{i}=\left(b_{i}, b_{i}^{\prime}, b_{i}^{\prime \prime}\right)$, then

$$
a_{i} \cap b^{\prime \prime}=a_{i} \cap b \cap a_{i} \cap b^{\prime}=a_{i} \cap\left(b_{1}, \cdots, b_{m}\right) \mathbf{n} a_{i} \cap \mathbf{n}\left(b_{1}^{\prime}, \cdots, b_{m}^{\prime}\right),
$$

whence by (L5), setting $c_{i}=\left(b_{1}, \cdots, b_{i-1}, b_{i+1}, \cdots, b_{m}\right)$, and $c_{i}^{\prime}$ and $c_{i}^{\prime \prime}$ equal to the corresponding dashed expressions, we have

$$
\left(a_{i} \mathbf{n} c_{i}^{\prime \prime}, b_{i}^{\prime \prime}\right)=\left(a_{i} \mathbf{\Lambda} c_{i}, b_{i}\right) \mathbf{n}\left(a_{i} \mathbf{n} c_{i}^{\prime}, b_{i}^{\prime}\right),
$$

whence, by strong coprimeness, after reduction, $b_{i}^{\prime \prime}=b_{i} \mathbf{n} b_{i}^{\prime}$.

That is, $B$ is a homeomorphic image of the subdirect product $B^{*}=B_{1} \hat{x} \cdots \hat{x} B_{n}$. But if $b_{i}=b_{i}^{\prime}$, and $b^{*}$ in $B^{*}$ is the image of $\left(b_{i}, b_{i}^{\prime}\right)$ of $B_{i}$, then $b^{*} \cap\left[b_{1}, \cdots, b_{n}\right]=b_{i} \neq b_{i}^{\prime}=b^{*} \cap\left[b_{1}^{\prime}, \cdots, b_{n}^{\prime}\right]$, whence, by (L1), $\left[b_{1}, \cdots, b_{n}\right] \neq\left[b_{1}^{\prime}, \cdots, b_{n}{ }^{\prime}\right]$, and the homeomorphism is an isomorphism. In summary, we have proved the following theorem.

Theorem 1. A given B-lattice $B$ (with largest element) is isomorphic with the subdirect product $B_{1}^{*} \cdots B_{n}^{*}\left(B_{i}^{*}\right.$ any B-lattice with largest element) if and only if $B$ contains strongly coprime sublattices $B_{1}, \cdots, B_{n}$ respectively isomorphic with $B_{1}^{*}, \cdots, B_{n}{ }^{*}$ such that any $b \in B$ can be expressed as a meet $\left(b_{i_{1}}, \cdots, B_{i_{m}}\right)$, where $m$ is finite and $b_{i_{k}} \in B_{i_{k}}$.

Notice that if $n$ is finite, then a subdirect product is a direct product; while if $n=2$, then strong coprimeness is equivalent to coprimeness.

4. Uniqueness Theory. Let $L$ be any lattice (with a largest element), and suppose that $L$ is isomorphic with two subdirect products $A_{1} \hat{x} \cdots \hat{x} A_{m}$ and $B_{1} \hat{x} \cdots \hat{x} B_{n}$. We know by the sec-

$\dagger$ The identical relations (L2)-(L5) can be checked seriatim. 
ond paragraph of $\S 3$, how to identify the $A_{i}$ (and $B_{i}$ ) with strongly coprime sublattices of $L$ in such a way that any element of $L$ can be represented as the meet of a finite number of elements of the various $A_{i}$ (or $B_{i}$ ). The reader can easily check the statement that, since $\left(a_{i}, a_{j}\right)=b_{k},\left(b_{k} \in B_{k}\right)$, if and only if $a_{i} \in B_{k}$ and $a_{j} \in B_{k}$, each $B_{i}$ is the subdirect product of its intersections with the various $A_{i}$; this proves the following statement.

Theorem 2. If $L=A_{1} \hat{x} \cdots \hat{x} A_{m}=B_{1} \hat{x} \cdots \hat{x} B_{n}$ is any lattice, $\uparrow$ then $L=F_{1,1} \hat{x} \cdots \hat{x} F_{m, n}$, where $A_{i}=F_{i, 1} \hat{x} \cdots \hat{x} F_{i, n}$ and $B_{j}=F_{1, j} \hat{x} \cdots \hat{x} F_{m, j}$.

Corollary 1. A lattice has at most one expression as a subdirect product of factors not themselves subdirect products.

Corollary 2. A finite lattice has a unique expression as the direct product of lattices not themselves direct products of lattices with fewer elements. The factors of any expression of the lattice as a direct product are direct products of the factors of this special decomposition into prime factors.

These corollaries are of extremely general application. $\ddagger \mathrm{We}$ now assume in addition that $L$ satisfies the following postulate.

$(\phi)$ Any sequence $a_{1}, a_{2}, a_{3}, \cdots$ of elements of $L$, such that $a_{k}<a_{k+1}$ for every $k$, is finite.

Well-order the expressions $L=L_{1}{ }^{i} \hat{x} \cdots \hat{x} L_{n}{ }^{i}$ of $L$ as a subdirect product, and apply Theorem 2 iteratedly. If we concentrate our attention on the corresponding well-ordered set of meets $\left(a_{1}{ }^{i}, \cdots, a_{\alpha_{i}}{ }^{i}\right)=a$ representing a fixed $a \in L$ (each $a_{h}{ }^{i}$ lying in just one of the $L_{k}{ }^{j}$ for each $j \leqq i$, by Theorem 2), we see that the expression $\left(a_{1}{ }^{i}, \cdots, a_{\alpha_{i}}{ }^{i}\right)$ undergoes $\S$ in virtue of $(\phi)$ at most a finite number of transmutations. Hence we can proceed through limit-numbers, and, by transfinite induction, we have the following result.

$\dagger$ By definition of subdirect product, either $m=n=1$ and the theorem is trivial, or the $A_{i}, B_{j}$, and $L$ have largest elements.

$\ddagger$ See Theorem 3.1 of the author's paper On the combination of subalgebras, Proceedings of the Cambridge PhilosophicalSociety, vol. 29 (1933), pp.441-464. This article will be cited in future references as "Subalgebras."

$\S$ Each transmutation replaces an $a_{h}{ }^{i}$ by the meet of $a_{h^{\prime}}{ }^{i+1}>a_{h}{ }^{i}$ and $a_{h^{\prime \prime}}{ }^{i+1}>a_{h^{i}}$. 
Theorem 3. A lattice satisfying $(\phi)$ has one and only one expression as a subdirect product of factors not themselves subdirect products.

Theorem 3 can evidently be applied to the ideals in rings which satisfy the ideal-chain theorem.

5. Standard Exceptions to (L6). Let $B$ be any $B$-lattice, suppose $g_{1}, g_{2}$, and $g_{3}$ to be any three elements of $B$, and refer to Tables I-III of "Subalgebras"-only replacing $A_{i}, B_{i}, M_{i}, N_{i}$, $C_{i}, F_{i}$, and $H_{i}$ by $a_{i}, b_{i}, m_{i}, n_{i}, c_{i}, f_{i}$, and $h_{i}$.

Suppose $c_{i}=c_{j}$ for some $i \neq j$. Then $a=\left(c_{i}, c_{j}\right)=c_{\imath} \cap c_{j}=b$, whence $\left(g_{1}, h_{1}\right)=\left(g_{1}, h_{1}, h_{2}, h_{3}\right)=\left(g_{1}, f_{1} \cap f_{2} \cap f_{3}\right)=\left(f_{2} \cap f_{3}\right) \cap\left(g_{1}, f_{1}\right)$ [by (L5) ] $=f_{2} \cap f_{3} \cap f=f_{2} \cap f_{3}$, which is to say, $\left(g_{1}, g_{2}, \cap g_{3}\right)=\left(g_{1}, g_{2}\right)$ $\boldsymbol{n}\left(g_{1}, g_{3}\right)$. If therefore (L6) is violated at all, we must have some instance where the $c_{k}$ are all distinct, yet $\left(c_{i}, c_{j}\right)=a$ and $c_{i} \mathbf{n} c_{j}=b$ for $i \neq j$, whence $\left(c_{1}, c_{2} \boldsymbol{\Pi} c_{3}\right) \neq\left(c_{1}, c_{2}\right) \mathbf{n}\left(c_{1}, c_{3}\right)$. This proves the following fact.

Theorem 4. If a B-lattice is not a C-lattice, it contains a sublattice of order five and fixed structure not a $C$-lattice.

Combining Theorem 4 with the result, due to Dedekind, $\dagger$ that any lattice not a $B$-lattice contains a sublattice of order five and fixed structure not a $B$-lattice, we get the following result.

Corollary. If a lattice is not a C-lattice, it contains a sublattice of order five which is not a C-lattice.

6. Specialization by Induction. Suppose $B$ of $\$ 5$ satisfies condition $(\phi)$ of $\S 4$, and consider the exception referred to in Theorem 4 . We can by $(\phi)$ choose $c_{1}^{*} \supset c_{1}$ covered by $b$ (see $\$ 2$ ). Theorems 8.1 and 9.1 of "Subalgebras" show us successively that $c_{3}$ covers $\left(c_{1}^{*}, c_{3}\right), b=c_{2} \cap c_{3}$ covers $c_{2}{ }^{*}=c_{2} \boldsymbol{\Pi}\left(c_{1}^{*}, c_{3}\right)$, hence $c_{1}{ }^{*}$ and $c_{2}{ }^{*}$ both cover $a^{*}=\left(c_{1}^{*}, c_{2}^{*}\right)$. Similarly $b=c_{3} \boldsymbol{\Pi} c_{2}^{*}$ covers $c_{3}^{*}=c_{3} \boldsymbol{\Pi}\left(c_{1}^{*}, c_{2}^{*}\right)$, and, since $c_{3}^{*} \supset a^{*},\left(c_{1}^{*}, c_{3}^{*}\right)=\left(c_{2}^{*}, c_{3}^{*}\right)=a^{*}$. This proves the following theorem.

THEOREM 5. If $B$ is any B-lattice satisfying $(\phi)$, then either $B$ is a C-lattice or we can find a sublattice of $B$ consisting of a least element $a^{*}, c_{1}^{*} \neq c_{2}{ }^{*} \neq c_{3}^{*} \neq c_{1}^{*}$ covering $a^{*}$, and $b=c_{1}^{*} \cap c_{2}^{*}=c_{2}{ }^{*} \cap c_{3}{ }^{*}$ $=c_{3}^{*} \cap c_{1}^{*}$ covering $c_{1}^{*}, c_{2}^{*}$, and $c_{3}^{*}$.

† Gesammelte Werke, 1931, vol. II, p. 255. 
7. Facts about Ideals. Throughout, $R$ will be understood to denote a commutative ring which has a principal unit $l$ and satisfies the Basis Theorem. Our notation will be that of van der Waerden $\dagger$ except that we shall denote by $(A, B)$ the 1.c.m., and by $A \cap B$ the g.c.f., of any two given ideals $A$ and $B$. This is the inverse of van der Waerden's notation.

The following are either known or immediate corollaries of known results:

(1). The only ideals in $R$ are $R$ and 0 if, and only if, $R$ is a field.

(2). If $I$ is a largest ideal in $R$, then $0: I$ is a least ideal if and only if it is a principal ideal.

(3). Any ideal I covered by $R$ is a prime ideal.

8. Application of Theorem 1. On the basis of Theorem 1 , it is possible to reconstruct the combinatorial theory of an important class of ideals.

By an ideal of genus 1 will be meant any ideal $I$ which contains an appropriate finite product $P_{1}{ }^{n_{1}} \cdots P_{\omega}{ }^{{ }^{\omega}}$ (where $P_{i}$ denotes any ideal covered by $R$ ). We shall prove the following result.

TheOREM 6. The ideals of genus 1 in $R$ are a B-lattice, which is the subdirect product of the sublattices $\mathfrak{P}_{1}, \mathfrak{P}_{2}, \mathfrak{P}_{3}, \cdots$ of the primary ideals under the ideals $P_{1}, P_{2}, P_{3}, \cdots$ covered by $R$.

That they are a lattice of which the $\mathfrak{B}_{i}$ are sublattices is obvious, while that they are a $B$-lattice follows from Theorem 27.1 of "Subalgebras."

But the $\mathfrak{P}_{i}$ are strongly coprime, since if $Q_{1}, \cdots, Q_{m}$ satisfy the relation $Q_{k} \notin P_{i}$ for every $k$, and $Q$ is primary under $P_{i}$, then $Q \cap\left(Q_{1}, \cdots, Q_{m}\right)=R$, being contained in no ideal covered by $R$. And by a theorem of $\mathrm{E}$. Noether, any ideal can be expressed as the meet of a finite number of primary ideals. Theorem 6 is now merely a translation of Theorem 1 in terms of ideals.

9. Application of Theorem 5. It is not difficult to show from known results the following theorem.

Theorem 7. If $R$ contains a largest ideal $I$, and another ideal $A \subset I$ for which $(A: I) / A$ is not a principal ideal, then the ideals of $R$ are not a $C$-lattice.

$\dagger$ Moderne Algebra, 1930-31; especially vol. 2, Chap 12, in which will be found the Basis and Ideal-chain Theorems. 
For since $l$, commutativity, and the Basis Theorem are preserved under homeomorphism, we can assume $A=0$; while by (2) we can assume $(0: I)$ is not a least ideal.

By the Ideal-chain Theorem we can further choose a largest subideal $J>0$ in $0: I$, and then $x \notin J, y \notin R x$ satisfying $y \in J$, and $w=x+y$. But a second homeomorphism permits us to assume $(R x, R y)=0$, yet $x \neq 0, y \neq 0$. This makes it obvious that $(R x, R y) \cap R w \neq(R x \cap R w, R y \cap R w) \exists x$.

Conversely, suppose the ideals of $R$ are not a $C$-lattice. By Theorem $5, R$ has a homeomorphic image $R^{*}$ which contains three least ideals $A \neq B \neq C$ such that $A \cap B=B \cap C=C \cap A$.

Consider $R^{*} /(0: A)$; it is a field, whence, by (1), $0: A$, and similarly $0: B$ and $0: C$, are largest ideals $\dagger$ in $R$. For if $r a \neq 0$ $\left[a \in A, r \in R^{*}\right]$, then $r a \in A$ generates $A$; consequently $r^{-1}$ exists such that $r^{-1} r a=a$ and $r^{-1} r \equiv l(0: A)$.

Again, if $0 \neq b \in B \subset A \cap C$, then $b=a+c$, where (since $a=0$ or $b=0$ would imply $B=C$ or $B=A) a \neq 0, c \neq 0$. And since $(A, C)=0,0=r b=r(a+c)=r a+r c$ implies $r a=r c=0$. Consequently $0: B \subset(0: A, 0: C)$, and $0: A=0: B=0: C=I$, where $I$ is a largest ideal in $R^{*}$, yet, by (2), $0: I$ is not a principal ideal in $R^{*}$. Referring back to the corresponding ideals in $R$, we see that $R$ does not satisfy the conclusions of Theorem 7 .

We can combine Theorem 7, its converse, and Theorem 25.2 of "Subalgebras" in the following theorem.

THEOREM 8. For the ideals of $R$ to be isomorphic (with respect to l.c.m. and g.c.f.) with a system of point sets (with respect to sum and product), it is necessary and sufficient that if $I$ is any largest ideal in $R$, and $A \subset I$ another ideal, then $(A: I) / A$ is a principal ideal in $R / A$.

It is a corollary that the identity $A:(A: Q)=(Q \cap A)$ upon ideals is a sufficient condition for distributive combination.

HARVARD UNIVERSITY

$\dagger R=0: A$ is of course excluded since $l \$ 0: A$. 